\title{
Dead Zones and the Origin of Planetary Masses
}

\author{
Soko Matsumura \& Ralph E. Pudritz, \\ Department of Physics \& Astronomy, McMaster University, \\ Hamilton, ON, L8S 4M1, Canada \\ soko@physics.mcmaster.ca,pudritz@physics.mcmaster.ca
}

\begin{abstract}
Protoplanets accrete material from their natal protostellar disks until they are sufficiently massive to open a gap in the face of the disk's viscosity that arises from the magneto-rotational instability (MRI). By computing the ionization structure within observationally well-constrained disk models, we demonstrate that poorly ionized, low viscosity "dead zones" stretch out to 12 AU within typical disks. We find that planets of terrestrial mass robustly form within the dead zones while massive Jovian planets form beyond. Dead zones will also halt the rapid migration of planets into their central stars. Finally, we argue that the gravitational scattering of low mass planets formed in the dead zone, to larger radii by a rapidly accreting Jupiter beyond, can explain the distribution of planetary masses in our solar system.
\end{abstract}

Subject headings: planetary systems: formation - solar system: formation - planets: general - accretion disks - MHD - stars: pre-main-sequence

\section{Introduction}

Current models for planet formation suggest either that Jovian planets are formed through gas accretion onto cores with $\sim 10$ Earth masses that are themselves assembled out of planetesimals (Mizuno 1980; Pollack et al. 1996), or through gravitational collapse when protostellar disks become cold and/or dense enough to be locally gravitationally unstable (Boss 1997; Mayer et al. 2002). However, independent of how their protoplanetary cores were formed, planets must open gaps in their natal disk before they become isolated. Ultimately, it is the process of gap-opening that terminates their growth or at least severely restricts their final masses. A planet must be sufficiently massive to tidally open a gap in the face of the disk's viscosity which acts to fill in a forming gap (Lin \& Papaloizou 1993; Ida \& Lin 2004). 
In this Letter, we calculate the gap-opening masses within protostellar disk models that are well constrained by the observations and analyzed in Matsumura \& Pudritz (2003) (MP03), and show that there should be a distinct break in planetary masses akin to the difference between terrestrial and Jovian planetary masses. The reason for this break is that protostellar disks are likely to contain regions of very low viscosity - dead zones - in which the planetary gap-opening mass is much smaller than in regions of normal viscosity. In $\S 2$, we lay out the theory of gap-opening masses in turbulent disks. We updated our MP03 disk models to include the effect of a nearby OB star, additional ionization sources, dust grains, as well as the turbulence driven in a dead zone by active layers (§3) and find that terrestrial mass planets form within the dead zones, while gas giant planets form outside of them $(\S 4)$. Finally, in $\S 5$, we apply our results to show that dead zones can halt the rapid inward migration of protoplanets and that scattering of low mass planets in the dead zone to larger disk radii by a rapidly accreting gas giant beyond, could explain the structure of our own solar system.

\section{Gap-opening masses and disk viscosity}

The minimum gap-opening mass in a fully turbulent region of a protostellar disk, $M_{p, t u r b}$, is attained when the angular momentum transport rate by a disk's turbulent viscosity becomes equal to that by a planetary tidal force (Lin \& Papaloizou 1993). It is readily shown that the gap-opening mass depends only on a disk aspect ratio $h / r_{p}$ (h is the local pressure scale height of a disk and $r_{p}$ is an orbital radius of the planet) and the turbulent viscosity parameter $\alpha_{\text {turb }}$. For the case that the disk's turbulence is absent, we applied the minimum gap-opening mass $M_{p, d a m p}$ obtained by Rafikov (2002). Lin \& Papaloizou (1993) also calculated a gap-opening mass in an inviscid disk by assuming that the density waves shock and damp immediately, but this requires a rather large planetary mass - the mass inside the Hill radius $r_{H}=h$. Rafikov (2002) showed that the density waves excited by a much smaller planet eventually shock and lead to the gap formation. The ratio of the gap-opening planetary mass for a region that is fully turbulent, compared to that for an inviscid disk, can be written as

$$
\frac{M_{p, t u r b}}{M_{p, \text { damp }}} \geq 2.5 \sqrt{\alpha_{\text {turb }}}\left(\frac{h}{r_{p}}\right)^{-23 / 26} Q^{5 / 13},
$$

in all cases relevant to our disk model; where $Q=\Omega c_{s} /(\pi G \Sigma)$ is the Toomre parameter that measures the gravitational stability of the disk, $\Omega$ is the Keplerian angular velocity, and $\Sigma$ is the surface mass density. For standard parameters, the critical mass ratio is of the order $M_{p, t u r b} / M_{p, \text { damp }} \sim 100$ which is comparable to the mass difference between terrestrial and Jovian planets. 
A major source of a disk's viscosity is thought to be hydromagnetic turbulence that is driven by the magneto-rotational instability (MRI) (Balbus \& Hawley 1998). This mechanism requires good coupling between the partially ionized gas of the disk and the magnetic field. Poor coupling, which occurs when this gas is not ionized sufficiently, leads to the formation of a so-called dead zone wherein the MRI disk viscosity is effectively zero. The spatial extent of a dead zone has been calculated by many authors (Gammie 1996; Sano et al. 2000; Glassgold et al. 2000; Fromang et al. 2002; Matsumura \& Pudritz 2003). Physically, the size of a dead zone can be determined by the condition that the local growth rate of the MRI turbulence $\left(\simeq V_{A} / h\right)$ becomes smaller than the Ohmic diffusion rate $\left(\simeq \eta / h^{2}\right)$ where $\eta$ is the magnetic diffusivity, and $V_{A} \equiv B / \sqrt{4 \pi \rho} \sim \alpha_{\text {turb }}^{1 / 2} c_{s}$ is the Alfvén speed ( $\rho$ is the mass density, $B$ is the magnetic flux density, and $c_{s}$ is the sound speed). Detailed numerical calculations show that this occurs when the ratio of these two growth rates - known as the magnetic Reynolds number:

$$
R e_{M} \equiv \frac{V_{A} h}{\eta}
$$

is less than a critical value of $R e_{M \text {,crit }}=10^{2}-10^{4}$ (Fleming et al. 2000). We use $R e_{M \text {,crit }}=$ $10^{2}-10^{4}$ and $\alpha_{\text {turb }}=10^{-3}-1$ in this paper ${ }^{1}$. Since the magnetic diffusivity $\eta$ is inversely proportional to the electron fraction, the MRI turbulence tends to be absent in poorly ionized regions of the disk.

In the surface layers of a disk, MRI turbulence will always be present and can drive vertical oscillations into the dead zone below. This process leads to angular momentum transport whenever the dead zone is not significantly denser than the active layers: $\Sigma_{\mathrm{DZ}} / \Sigma_{\mathrm{AL}} \leq 10$ (Fleming \& Stone 2003), where $\Sigma_{\mathrm{DZ}}$ and $\Sigma_{\mathrm{AL}}$ are the surface mass density of the dead zone and the active layers respectively. Therefore, the "true" dead zone should satisfy both conditions and we calculated it using a self-consistent model for the disk structure.

\section{Disk Model}

Most stars are thought to be formed in star clusters such as the Orion nebula cluster. A protostellar disk in such an environment will be irradiated by nearby luminous $\left(10^{5}-10^{6} L_{\odot}\right)$ OB stars in the cluster. At the outer part of the protostellar disk, the disk heating by the UV radiation from a central T-Tauri star (TTS) is overwhelmed by the combined radiation field of the nearby OB stars. As the outer disk temperature increases, the disk flares more strongly and therefore planets are expected to have a higher gap-opening mass. Recently, Robberto et al. (2002) (RBP02) improved the self-consistent model of passive protostellar disks of Chiang \& Goldreich (1997) by taking into account the effect of an external OB star. They showed that the disk aspect ratio changes significantly. We used their model to 
calculate the vertical structure of a disk around a TTS which is exposed to OB stars ${ }^{2}$.

Following RBP02 and without loss of generality, we assume that a disk is oriented faceon with respect to an $\mathrm{O}$ star which is located at a typical distance of $0.1 \mathrm{pc}$ from the disk and has the luminosity of $L_{s}=6 \times 10^{38} \mathrm{erg} \mathrm{s}^{-1}$ and a Strömgren radius of $R_{\mathrm{HII}}=10^{18} \mathrm{~cm}$. In all of our models, we adopt the disk surface mass density at $1 \mathrm{AU}$ of $\Sigma_{0}=10^{3}$ or $10^{4} \mathrm{~g} \mathrm{~cm}^{-2}$ which is equivalent to a disk mass inside $100 \mathrm{AU}$ of $\sim 0.01$ or $0.1 M_{\odot}$ respectively $\left(M_{\odot}\right.$ is the mass of the Sun). These disk masses are typical for T Tauri stars (Hartmann et al. 1998; Kitamura et al. 2002).

The electron fraction that controls the magnetic diffusivity of disks, is determined by the balance between ionization and recombination. For the ionization sources, we include $\mathrm{X}$-rays from the central star and a nearby $\mathrm{O}$ star, cosmic rays, radioactive elements as well as the thermal collisions of alkali atoms. Among those, X-rays from the central star and cosmic rays are the two major ionizing sources of protostellar disks (e.g. MP03). Protostellar X-rays may be generated within large loops of stellar magnetic field that result from reconnection during magnetospheric accretion, as shown in time-dependent calculations (e.g. Hayashi et al. 1996). We assume that magnetic loops extend out to distances $(r, z)=\left(2 R_{*}, 2 R_{*}\right)$ from the stellar surface and adopt a typical T Tauri star's X-ray luminosity $L_{x}=10^{30} \mathrm{erg} \mathrm{s}^{-1}$ and temperature $k T_{x}=2 \mathrm{keV}$ (Feigelson, private communication). We also assume that cosmic rays propagate along field lines and that the strength of cosmic rays is constant across the disk surface. Both ionization rates as well as the uniform ionization by radioactive elements: $\xi_{\mathrm{RA}}=6.9 \times 10^{-23} \mathrm{~s}^{-1}$ are calculated as in MP03. In this paper, we also include the thermal ionization of alkali metals (potassium) due to heating by the central star following Fromang et al. (2002). They showed that there is a magnetically active zone at the innermost radii $(r \lesssim 0.1 \mathrm{AU})$. We find, however, that the thermal ionization effect is killed if we take account of the recombination on grains (see below). Our dead zone stretches from the inner disk radius to a few tens of $\mathrm{AU}$. We also find that the X-ray ionization by a fiducial nearby O star with the X-ray luminosity $L_{x}=10^{34} \mathrm{erg} \mathrm{s}^{-1}$ and temperature $k T_{x}=2 \mathrm{keV}$ is too weak to affect the ionization structure of the disk.

For recombination processes, we considered the reactions among electrons, molecular ions, metals and grains. At disk density $n \geq n_{\text {crit }} \sim 10^{12} \mathrm{~cm}^{-3}$, grains are very effective at reducing the charge in disks and hence increasing the diffusivity $\eta$. Were it not for the stimulation of turbulence in the body of the disk from the envelope, our detailed calculations, which followed the method by Umebayashi \& Nakano (1990) and Fromang et al. (2002), show that very extensive dead zones - out to $16-29 \mathrm{AU}$ - are to be expected ${ }^{3}$. However, this "stimulated" turbulence limits the extent of a dead zone to smaller radii, typically $12-25$ AU for our two fiducial disk column densities. 


\section{Results}

We show the spatial structure of disks as well as their internal dead zones in Fig. 1 and 2 which correspond to disk column densities of $\Sigma_{0}=10^{3}$ and $10^{4} \mathrm{~g} \mathrm{~cm}^{-2}$ respectively. Three curves show the disk height $z_{\text {rem }}$ where the magnetic Reynolds number $R e_{M}$ reaches its critical value $10^{3}$ for $\alpha_{\text {turb }}=0.1,0.01$, and 0.001 from inside to outside. Also shown is the disk height $z_{\text {surf }}$ where the surface mass density ratio of below and above it is equal to $10 ; \Sigma_{\text {below }} / \Sigma_{\text {above }}=10$ (a dashed line). We define the dead zone as the region where $z \leq z_{\text {rem }}$ and $z_{\text {rem }}>z_{\text {surf }}$ - the intersection of $z_{\text {rem }}$ and $z_{\text {surf }}$ marks the outer dead zone radius As the magnetic field becomes stronger (the parameter $\alpha_{\text {turb }}$ becomes larger), the dead zone becomes smaller. For a standard disk $\left(\Sigma_{0}=10^{3} \mathrm{~g} \mathrm{~cm}^{-2}\right)$ with $\left(R e_{M}, \alpha_{\text {turb }}\right)=\left(10^{3}, 0.01\right)$, we find that the dead zone stretches from the inner edge of a disk to $12 \mathrm{AU}$ and encompasses the terrestrial planet region in our solar system $(0.3-2 \mathrm{AU})$. For a moderately heavy disk $\left(\Sigma_{0}=10^{4} \mathrm{~g} \mathrm{~cm}^{-2}\right)$, the dead zone radius becomes $25 \mathrm{AU}$. These results agree well with previous works (e.g. Sano et al. 2000). It may be possible that cosmic rays do not ionize disks because they are swept away by disk winds. In this case, disk ionization is determined mainly by X-rays from the central star and an external star. The dead zone radii then become slightly larger (14 AU for $\Sigma_{0}=10^{3} \mathrm{~g} \mathrm{~cm}^{-2}$ and $36 \mathrm{AU}$ for $\Sigma_{0}=10^{4} \mathrm{~g} \mathrm{~cm}^{-2}$ ).

In Fig. 3 and 4, we show the calculated gap-opening masses of planets for disks with $\Sigma_{0}=10^{3}$ and $10^{4} \mathrm{~g} \mathrm{~cm}^{-2}$ respectively. Both disks are gravitationally stable (we find $Q_{\min }>3$ in our $\Sigma_{0}=10^{4} \mathrm{~g} \mathrm{~cm}^{-2}$ model) so that planet formation by gravitational instability should not occur in these disk models. The upper parallel horizontal lines show the gap-opening masses with various viscous parameters while the bottom solid horizontal line shows the gap-opening masses in an inviscid disk. Vertical lines with crosses indicate the extent of a dead zone. A thick solid line shows the fiducial gap-opening mass throughout the disk.

In the core accretion scenario, the minimum gap-opening mass inside the dead zone is dictated by wave damping (the lowermost line), while outside, the minimum gap-opening mass is determined by the strength of the MRI viscosity and hence the strength of the magnetic field (the value of $\alpha_{\text {turb }}$ ). The predicted planetary mass therefore makes a distinct jump upwards to a value determined by the value of $\alpha_{\text {turb }}$ outside the dead zone. Our fiducial cases, shown in Fig. 3 and 4 with a thick solid line, corresponds to $\alpha_{\text {turb }}=0.01$

outside the dead zone. This $\alpha_{\text {turb }}$ value is suggested by observations of protostellar disks on scales $\sim 10-100 \mathrm{AU}$ (Hartmann et al. 1998; Kitamura et al. 2002). Along this line in Fig. 3, we find the gap-opening mass becomes equal to an Earth mass $M_{E}$ at $\sim 0.7 \mathrm{AU}$ and a Jupiter mass $M_{J}$ at $\sim 17 \mathrm{AU}$. Our results show that Jupiter or more massive gas giant planets must form outside the dead zones while terrestrial and perhaps even ice giant planets (see below) are likely to form within them. 


\section{An integrated picture for solar system formation}

Our results show that massive planets in our fiducial disk models form beyond $12 \mathrm{AU}$. This further supports the need of planet migration as an explanation of the observed exosolar systems. The presence of a dead zone may solve a nagging problem of migration theory there is no general mechanism of halting a planet's migration into the central star. The standard migration picture consists of two types of migration. When a protoplanet is not very massive, it migrates through the disk without opening a gap (type I). As a protoplanet gains a sufficient mass, it opens a gap in the disk and subsequently migrates with the disk on a viscous timescale (type II). The type I migration is roughly two orders of magnitudes faster than the type II migration (Ward 1997; Terquem 2003). This means that in an inviscid region like a dead zone, a migrating planet will be stalled as soon as it opens a gap (Chiang et al. 2002). Since the presence of finite dead zones is a robust feature of the protostellar disks, they may act as natural barriers that prevent the rapid loss of planets into their central stars.

The planetary masses predicted by our disk model (and others) increase with disk radius. In our solar system, this is not observed - the lower mass ice giants Uranus and Neptune are found at larger radii (19.2 and 30.1 AU respectively). One scenario which can explain this mass sequence is the photoevaporation of the disk that reduces the surface mass density significantly beyond $\sim 15$ AU within $\sim 10^{7}$ years (Adams et al. 2004) but this may be too long for currently accepted disk lifetimes.

We suggest an alternative - the gravitational scattering of lower mass protoplanets from within the dead zone to much larger radii by a gas giant located just outside of it. Numerical experiments (Thommes et al. 1999) have shown that a rapidly accreting Jupiter can scatter a more slowly growing protoplanetary core on an interior orbit as the former's Hill sphere expands. The scattered low mass body will ultimately circularize its orbit by dynamical friction at sufficiently large disk radii to be decoupled from the scatterer. This scenario has the distinct advantage of building the cores of ice giants much faster because it happens in the inner region of the disk. This process may occur naturally in our disk model. For example, in Fig. 3 along the curve $\left(R e_{M}, \alpha_{\text {turb }}\right)=\left(10^{3}, 0.01\right)$, we find that a $0.74 M_{J}$ planet is formed just outside the dead zone at $r=12 \mathrm{AU}$, while the maximum mass just inside the dead zone is $0.08 M_{J}$ - which is roughly equal to the mass of an ice giant planet. The inner core(s) would be scattered when the disk gas becomes sufficiently tenuous so that the eccentricity can be excited.

An inevitable consequence of a dead zone is that material from the well-coupled region beyond its outer edge will accumulate at this interface. The increasing column density of such an annulus may push the dead zone outward in radius, and may even become gravitationally 
unstable. We are currently investigating these time-dependent problems.

In conclusion, we have calculated planetary gap-opening masses in standard mass $(\sim$ $\left.0.01 M_{\odot}\right)$ and moderately heavy $\left(\sim 0.1 M_{\odot}\right)$ disk models which are exposed to a nearby

$\mathrm{O}$ star. With widely accepted values of $\left(R e_{M}, \alpha_{\text {turb }}\right)=\left(10^{3}, 0.01\right)$, we have found that the dead zone stretches out to $12 \mathrm{AU}$ for the standard mass disk. We have shown that there is a distinct and model-independent range of planetary masses within the dead zone compared to the well-coupled zone beyond. With $\left(R e_{M}, \alpha_{\text {turb }}\right)=\left(10^{3}, 0.01\right)$, this corresponds to terrestrial mass planets $\left(2.4 \times 10^{-4}-8.3 \times 10^{-2} M_{J}\right)$ vs Jovian mass planets $\left(0.74-7.6 M_{J}\right)$. The robust nature of our results leads us to conclude that dead zones are typical in protostellar disks and may play a central role in determining the masses of planetary families as well as their fates.

We thank Eric Feigelson, David Hollenbach, Doug Lin, Edward Thommes and James Wadsley for stimulating discussions and an anonymous referee for a very useful report. S. M. is supported by a SHARCNET Graduate Fellowship while R. E. P. is supported by a grant from the National Science and Engineering Research Council of Canada (NSERC).

\section{REFERENCES}

Adams, F. C., Hollenbach, D., Laughlin, G., \& Gorti, U. 2004, ApJ, 611, 360

Balbus, S. A. \& Hawley, J. F. 1998, Reviews of Modern Physics, 70, 1

Boss, A. P. 1997, Science, 276, 1836

Chiang, E. I., Fischer, D., \& Thommes, E. 2002, ApJL, 564, L105

Chiang, E. I. \& Goldreich, P. 1997, ApJ, 490, 368

Chiang, E. I., Joung, M. K., Creech-Eakman, M. J., Qi, C., Kessler, J. E., Blake, G. A., \& van Dishoeck, E. F. 2001, ApJ, 547, 1077

Fleming, T. \& Stone, J. M. 2003, ApJ, 585, 908

Fleming, T. P., Stone, J. M., \& Hawley, J. F. 2000, ApJ, 530, 464

Fromang, S. ., Terquem, C., \& Balbus, S. A. 2002, MNRAS, 329, 18

Gammie, C. F. 1996, ApJ, 457, 355

Glassgold, A. E., Feigelson, E. D., \& Montmerle, T. 2000, Protostars and Planets IV (The University of Arizona Press, 2000) 
Hartmann, L., Calvet, N., Gullbring, E., \& D’Alessio, P. 1998, ApJ, 495, 385

Hayashi, M. R., Shibata, K., \& Matsumoto, R. 1996, ApJL, 468, L37+

Ida, S. \& Lin, D. N. C. 2004, ApJ, 604, 388

Kitamura, Y., Momose, M., Yokogawa, S., Kawabe, R., Tamura, M., \& Ida, S. 2002, ApJ, 581,357

Lin, D. N. C. \& Papaloizou, J. C. B. 1993, Protostars and Planets III (The University of Arizona Press)

Matsumura, S. \& Pudritz, R. E. 2003, ApJ, 598, 645

Mayer, L., Quinn, T., Wadsley, J., \& Stadel, J. 2002, Science, 298, 1756

Mizuno, H. 1980, Progress of Theoretical Physics, 64, 544

Pollack, J. B., Hubickyj, O., Bodenheimer, P., Lissauer, J. J., Podolak, M., \& Greenzweig, Y. 1996, Icarus, 124, 62

Rafikov, R. R. 2002, ApJ, 572, 566

Robberto, M., Beckwith, S. V. W., \& Panagia, N. 2002, ApJ, 578, 897

Sano, T., Miyama, S. M., Umebayashi, T., \& Nakano, T. 2000, ApJ, 543, 486

Terquem, C. E. J. M. L. J. 2003, preprint (astro-ph/0309175)

Thommes, E. W., Duncan, M. J., \& Levison, H. F. 1999, Nature, 402, 635

Umebayashi, T. \& Nakano, T. 1990, MNRAS, 243, 103

Ward, W. R. 1997, Icarus, 126, 261

This preprint was prepared with the AAS LATEX macros v5.2.

${ }^{1}$ Recent numerical simulations by Fleming et al. (2000) defined the magnetic Reynolds number as $\operatorname{Re}_{M}=$ $c_{s} h / \eta$ and determined the critical value in the range of $10^{2}-10^{4}$. In our definition of $R e_{M}=\alpha^{1 / 2} c_{s} h / \eta$, $\left(R e_{M}, \alpha_{\text {turb }}\right)=\left(10^{2}, 0.01\right)$ and $\left(10^{3}, 0.01\right)$ correspond to their $\operatorname{Re}_{M}=10^{3}$ and $10^{4}$ respectively.

\footnotetext{
${ }^{2}$ We compared three different grain models which give the emissivity difference and therefore the temperature difference of up to $\sim 40 \%$. Two of them are RBP02's models of Type I and Type II grains whose radius is $0.1 \mu \mathrm{m}$ and $0.02 \mu \mathrm{m}$ respectively. The other is the disk model by Chiang et al. (2001) with an external stellar radiation. This grain model gives the lowest temperature of all. We chose an intermediate
} 


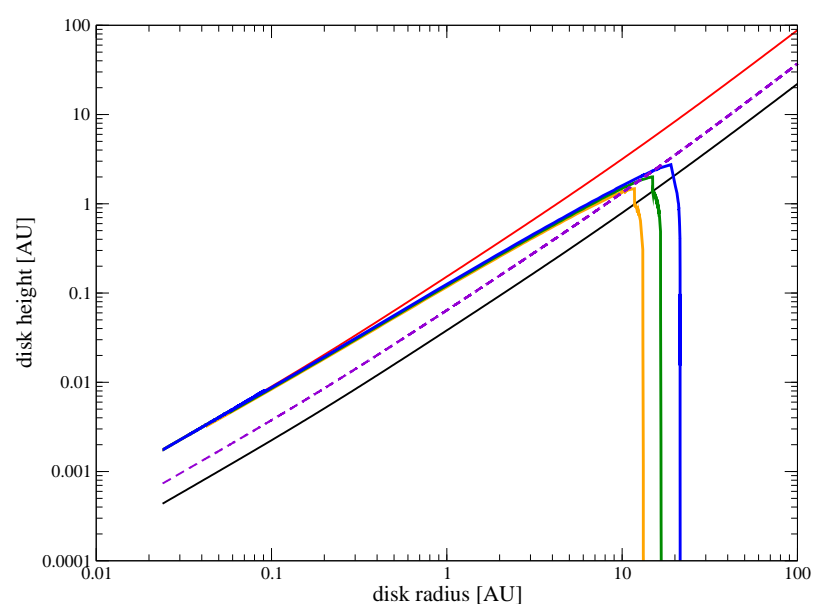

Fig. 1.- Dead zones calculated for the standard disk model with an external star. We used $\Sigma_{0}=10^{3} \mathrm{~g} \mathrm{~cm}^{-2}, L_{x}=10^{30} \mathrm{erg} \mathrm{s}^{-1}$, $k T_{x}=2 \mathrm{keV}$, and $R e_{M}=10^{3}$. The uppermost line shows the surface disk height; the lowermost line shows the pressure scale height while three curves show the dead zone boundaries for $\alpha=0.1,0.01$, and 0.001 from inside to outside. The dashed line is where $\Sigma_{\text {below }} / \Sigma_{\text {above }}=10$.

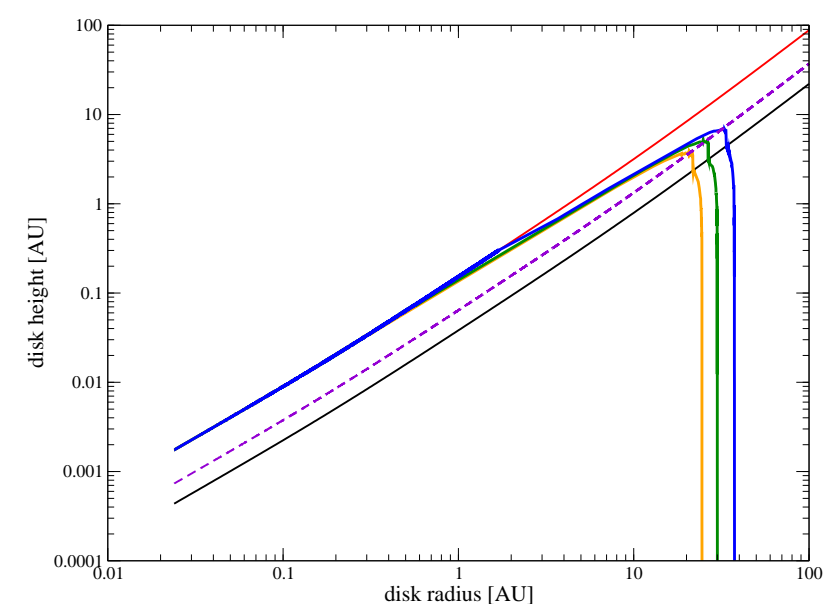

Fig. 2.- Dead zones calculated for a heavier disk of $\Sigma_{0}=10^{4} \mathrm{~g} \mathrm{~cm}^{-2}$ with an external star. For the explanation of each line, see Fig. 1.

model which uses the optical depth of $\tau_{\perp}=0$ and the type I grains of RBP02.

${ }^{3}$ To be consistent with the disk model, we assumed all grains have the same radius $0.1 \mu \mathrm{m}$. Our electron fraction for a solar nebula model is about 2 orders of magnitude larger than the one obtained by Sano et al. (2000) because we ignore grains of charge $\pm 2 e$. With their electron fraction and our choice of $R e_{M}=10^{3}$, the dead zone would be only slightly larger. 


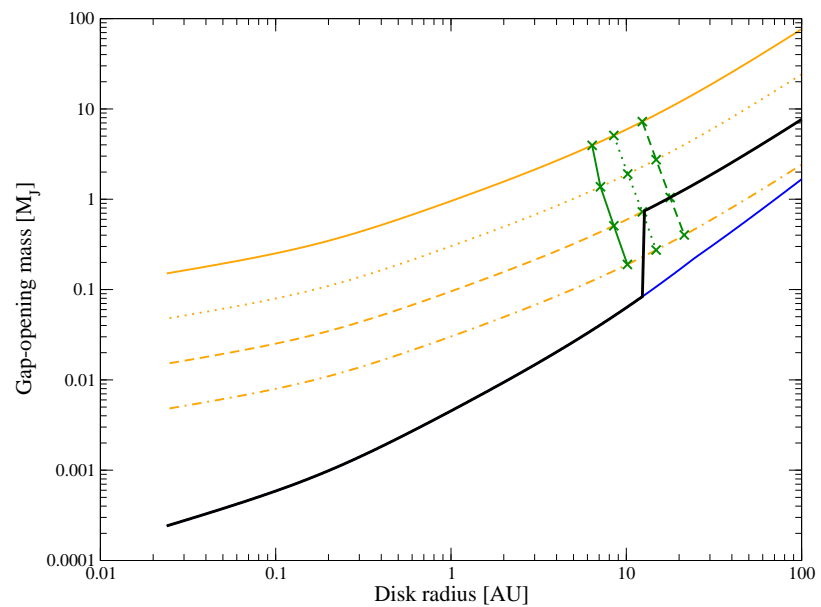

Fig. 3.- Gap-opening masses for a disk with the surface mass density, at $1 \mathrm{AU}$, of $\Sigma_{0}=10^{3} \mathrm{~g} \mathrm{~cm}^{-2}\left(\right.$ or $\left.M_{d} \sim 0.01 M_{\odot}\right)$. The lowermost line shows the gap-opening mass for the region with no MRI viscosity while the upper parallel lines show the gap-opening masses for a different strength of magnetic field: solid line is for $\alpha_{\text {turb }}=1$, dotted line is for 0.1, dashed-line is for 0.01, and dotdashed line is for 0.001. The dead zone for the magnetic Reynolds number: $R e_{M}=10^{2}$ is inside a solid line with crosses, that for $10^{3}$ is inside a dotted line with crosses, and that for $10^{4}$ is inside a dashed line with crosses. The thick solid line is our fiducial minimum gapopening mass throughout the disk for MRI "viscosity", $\alpha_{\text {turb }}=0.01$.

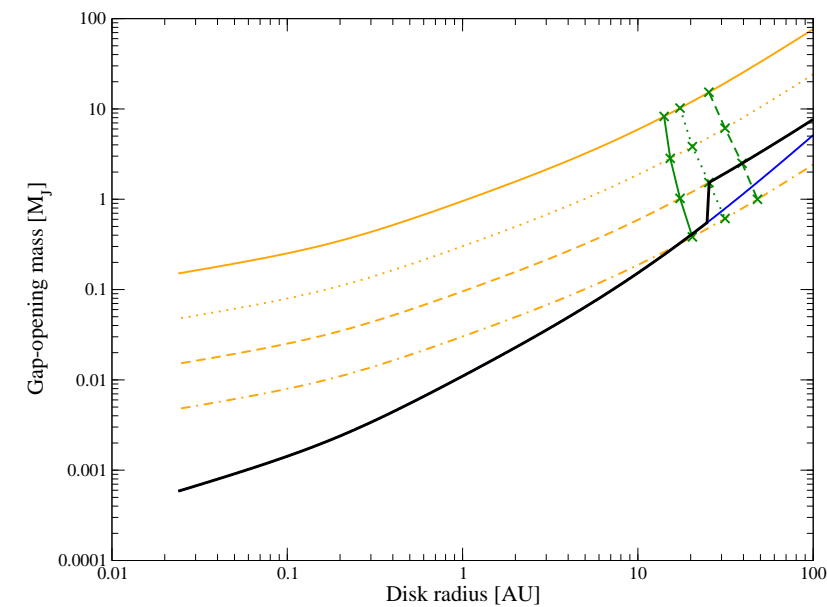

Fig. 4.- Gap opening masses for a disk with the surface mass density at $1 \mathrm{AU}$ of $\Sigma_{0}=10^{4} \mathrm{~g} \mathrm{~cm}^{-2}\left(\right.$ or $\left.M_{d} \sim 0.1 M_{\odot}\right)$. For the explanation of each line, see Fig. 3. 